\title{
Cooling System Rotor Dynamics in Military Combat Vehicle
}

\author{
Samo Zupan, Ivan Prebil ${ }^{\dagger}$, Brane Sirok and Marko Hočevar \\ Faculty of Mechanical Engineering, University of Ljubljana, Aškerčeva cesta 6, SI 1000 Ljubljana, Slovenia
}

(Received 12 April 2000; accepted 26 February 2002)

\begin{abstract}
To increase the power of the T 72 tank engine, it has been necessary to redesign the ventilator rotor of the cooling system. The basic mechanical loads have been analysed and the newly designed rotor has been tested with these loads. Dynamical properties have been determined at the different rotation speeds and with different bearing types and arrangements. Characteristic frequencies and transitional phenomena have been determined from the experimental data and the numerical analytical results. Boundary and initial conditions have been taken from the available experimental data and from relevant military standards. Numerical analysis has been carried out using finite element analysis software. In this paper the emphasis is on the analysis of the rotor-bearing system in the movable ventilator test stand housing. The results are presented in the form of transfer functions showing the dynamical properties of the rotor as a function of the excitation of the ventilator housing for different types of bearings and rotational speeds.
\end{abstract}

${ }^{\dagger}$ Member of the International Institute of Acoustics and Vibration (IIAV)

\section{INTRODUCTION}

Demands for increased power of the main (internal combustion) engine in combat vehicles require cooling systems with an increased heat dissipation capability. Such an increase in the cooling capability can be achieved only with a modification of the cooling fans, if the thermodynamic characteristics are to remain unchanged. At Turboinštitut in Ljubljana we have developed a new rotor for the cooling fan of the combat vehicles M-84 and T-72. ${ }^{1}$ While the integral geometric and kinematical properties remain the same, the new rotor causes an increase in the flow (Fig. 1). ${ }^{2,3}$ In the new design the aerodynamic loads have increased, the mechanical mass distribution has changed, and the maximum usable rotation speed has increased to $3500 \mathrm{rpm}$.

To study the resulting effects, we have made a numerical analysis of the fan rotor motion in its bearings. ${ }^{4,5}$ An experimental fan housing primarily intended for the measurements of flow parameters, was used for this experiment. A system adapted for dynamic excitation was intended to simulate the basic dynamics of the real system. We have measured the displacements (deformations) of the rotating rotor in relation to the moving fan housing. By comparing the results obtained with and without excitation we have determined the influence of the external mechanical disturbances on the rotational dynamics of the system. In the final phase, these results were used in the selection of a suitable rotor bearing arrangement in the fan housing.

\section{STRENGTH AND DYNAMIC ANALYSIS OF THE ROTOR}

For rotor analysis, we have chosen a load spectrum (profile) based on military standards (MIL) and requirements set by the military industry for comparison purposes. ${ }^{4,5}$ The loads of the rotor assembly originate in its mass (rotor, relief clutch analysis of the stress states in the rotor and an experimental

and shaft), amounting to almost $50 \mathrm{~kg}$, and are determined with expected amplitude levels of vibrations and impulses experienced during various operating conditions. As the largest load, according to MIL-STD-810D, we considered a short-term impulse in the radial and axial direction with a magnitude of $50 \mathrm{~g}$ (force amplitude $25 \mathrm{kN}$ ). Another important load is the centrifugal force caused by the rotor rotating at 1000 to $3000 \mathrm{rpm}$.

The strength analysis of the combat vehicle fan was carried with the finite element method using the computer program ANSYS 5.3. The model studied consisted of the base plate with fan blades and a retaining ring cast in Al-Mg alloy. The rotor is fixed with screw bolts to the steel flange of the relief clutch, which is manufactured in one piece with the shaft, and installed in the housing using a suitable bearing arrangement. The largest external fan loads are mainly the result of the accelerations of the tank hull caused by driving and shooting. The result is that maximum loads occur on the locations where the rotor is fixed to the relief clutch flange. The other parts of the fan (blades, and retaining ring) are not loaded at all in practice. The maximum von Misses stresses under external loads amount to approximately $5 \%$ of the stresses caused by the rotation at $3500 \mathrm{rpm}$. The numerical analysis showed that the critical location of the fan is the connection of blades to the retaining ring where von Misses stresses reach $154 \mathrm{~N} / \mathrm{mm}^{2}$ (Fig. 2). The technical elasticity limit of the Al-Mg alloy used is $168 \mathrm{~N} / \mathrm{mm}^{2}$.

During the testing of the modified rotors at rotational speeds lower than maximum, failures occurred with all the characteristics of brittle fracture of fan blades. The causes for such failures could, besides fatigue effects, be harmonic or randomly excited vibration of the rotor. Therefore, we made a modal analysis of the existing model comprising the fan rotor with the attached steel flange and the shaft with bearings connecting it to the housing. The model was fixed in all nodes of the mesh contacting the inner rings of the bearings and pre-stressed with the rotational speed effect of $3000 \mathrm{rpm}$. For the modal analysis we used the Guyan reduced mass ma-

International Journal of Acoustics and Vibration, Vol. 7, No. 3, 2002 (pp 185-189) 